\title{
Non-Stationary Temperature Behavior of Spindle Assemblies and Ball Screw Gears for High Precision Metal Cutting Machines
}

\author{
A.P. Kuznetsov ${ }^{1}$ \\ ${ }^{1}$ Moscow State Technological University "STANKIN", RU-127055, Moscow, Russia
}

\begin{abstract}
Typical temperature and thermoelastic models of structures of spindle units and ball screw systems of metal-cutting machines were considered. The features of determining the geometric and thermophysical parameters of these models were described in some detail. Both analytical and numerical solutions for estimating time-dependent temperatures are presented. The solutions were obtained for thermoelastic displacements of spindles and ball screw systems for various boundary conditions and limitations of deformations during heating due to the design of these elements of metal-cutting machines.
\end{abstract}

\section{Introduction}

Progress in improving the accuracy of metal-cutting machines is inextricably linked and is due to a deeper knowledge of thermal processes and effects occurring in machines needed to control them. Since 1950th till now researchers have performed a number of studies of thermal processes and effects that occur in metal-cutting machines during their operation. The conducted studies of accuracy indicators and machine properties give reason to believe that in the balance of machine accuracy, the share of temperature errors in the period of 1950 - 2020s changed from $20-30 \%$ to $70 \%[1,2]$. This follows from the analysis of energy requirements (energy barrier of accuracy), which are necessary and make it possible to achieve a certain level of processing accuracy (Fig.1) [3,4].

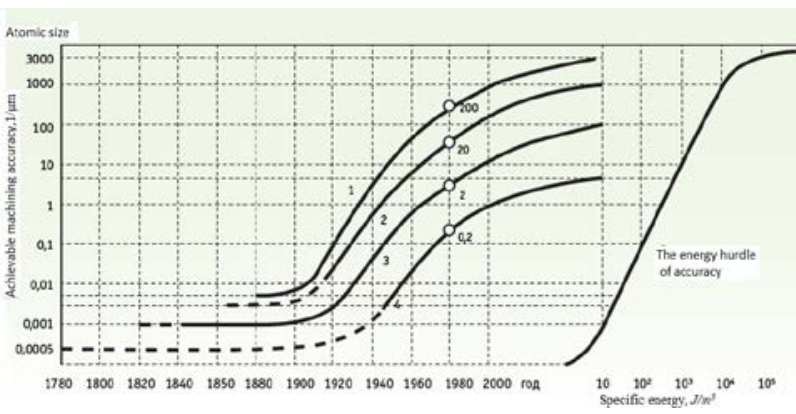

Fig.1. Evolution of the development of achievable machining accuracy of Machine tools:1-traditional, 2 - precision, 3 - high precision, 4-ultra-high precision

In the period of 1980-2020s, types and forms of methods for correcting and compensating the thermal behavior of forming elements of machine tools were developed [5,6], which are based on the use of linear and nonlinear regression methods or correlation between displacements and temperature of machine components and parts. These methods are based on direct methods for measuring temperature errors, indirect methods based on the measured temperature obtained from sensors (or without sensors) [7] located at various points of the machine and the subsequent determination of TCP offsets from the thermal model, indirect methods according to information received from sensors on different parameters (e.g., frequency of rotation, speed, axis, etc.), calculations based on the constructed or selected mathematical model, software-based methods such as thermal or thermo-elastic machine models, predictive models of changes of temperature deformations and machine control based on experimental data on the temperature behavior of the machine [8]. Over the past 30 years, the international organization for standardization (ISO) has developed several standards: ISO 230-3 (Thermal displacements in machine tools); ISO 10791-10 (Thermal displacements in machining centers); ISO 13041-8 (Thermal displacements in lathes), ISO 16907-2015(correction of geometric errors using $\mathrm{CNC}$ ), which define test procedures for evaluating thermal effects that lead to temperature deformation of the structural and layout structure of the machine and/or its positioning systems. The functional relationship between the measured temperature in the machine units and the amount of displacement can be established experimentally. With good repeatability and fairly stable reproducibility of the results, this functional relationship is expressed by an equation that can be used as the basis for the operation of the control device for compensation of thermal deformations [6,9-11]. Analysis of design and layout solutions of modern metal-cutting machines has shown that in the thermal model of the machine, the spindle assembly [9-11] and ball-screw systems [12,13] of movement of the working bodies of the machine are the main and dominant sources of the thermal instability. Since mathematical methods for describing thermal models [14-22], numerical and experimental stationary 
thermal conductivity methods, and FEM were most often used, non-stationary processes were either neglected or described using the simplest models of thermal balance without accounting for the full range of boundary conditions in determining temperatures and solving thermoelastic problems in a non-stationary temperature field. As a rule, this is due to the fact that for the accuracy required in the past, it was enough to consider the thermoelastic deformations of the spindle and drive screws as linear elongation of the rod in proportion to the average temperature. This paper presents solutions to non-stationary problems for determining the temperature fields of spindle assemblies and ball screw gears and their temperature deformations for conditions that limit their free movement to that in typical modern metalcutting machines.

\section{Thermal models of spindle units and ball screw units}

Analysis of the design of machine components and parts with an indication of the kinematic scheme, configuration of parts, their connections, material, identification of the main sources of heat, their type and location, heat exchange conditions and other factors are necessary for the formation of thermal models of the machine unit or part with specified and reasonable assumptions. The thermal model is the basis for creating a mathematical model written in the form of equations with boundary conditions that reflect the connections of a node or part in terms of their heat exchange. Mounting options used in machine tools, linear motion drive "lead screw-rolling nut", used in metal cutting machines, have four types of mounting supports of the lead screw and the first support always has a mounting of the lead screw and either allows or does not move the lead screw in the axial direction, and the second support is made in four versions: LI - the first and second supports support the lead screw with the possibility of free axial movement; LII - the second support only supports the screw, and the first does not allow you to move in the axial direction; LIII-the second support prevents axial movement of the lead screw; LIY - the second support is missing.

Despite all the variety of design features of building schemes for installing bearings in the front and rear spindle supports, which are determined by the requirements for providing the specified parameters and characteristics of spindle units (radial and axial stiffness, dynamic stiffness, rotation accuracy, spindle runout, speed parameters, required torque, durability, and others), existing spindle unit schemes can be divided into four fundamentally different types in relation to perception ( as direct, and reverse) axial loads of the front and rear spindle supports, and, accordingly, the ability to move in the axial direction:

SI-the rear support accepts the axial load in the forward and reverse directions, and the front support moves freely in the axial direction and accepts the radial load;

SII - the front support accepts the axial load in the forward and reverse directions, and the rear support moves freely in the axial direction and accepts the radial load;

SIII-the front and rear supports perceive the axial load in different directions, and one of the supports can move freely in the axial direction under the action of an external axial force;

SIY-the front and rear supports perceive the axial load in different directions, and one of the supports can move freely in the axial direction under the action of an internal axial force.

Thus, the analysis of structures and generalization of basic layout schemes for the construction of both spindle and ball screw units allows us to form a general temperature and thermoelastic models for calculating temperatures and axial displacements, which is due to and determined by the unity of models in the formulation and solution of this problem. Therefore, the thermal model of a part, unit or machine is defined as their geometric image, which adequately reflects the conditions of unambiguity due to the features of thermal processes that are formed by the functional purpose, technological and technical parameters, characteristics and properties under specified operating conditions.

The degree of adequacy of the thermal model is determined by the accepted simplifications, assumptions, and completeness of consideration and accounting in the thermal model of real thermophysical processes that are determined by the goal (s), tasks to be solved, and methods for achieving them.

If the criterion value $\mathrm{Bio}$ small $\mathrm{Bi}<0,1 \div 0,2$, in this case, the dimensionality of the problem decreases as the thermal resistance of the boundary layer is substantially more thermal resistance and the problem in finding the temperature on the surface of the part and any other point of the surface, and the temperature of the part at the Ld almost identical.

So, in table.1. the limits of the values of the smallest size Ld of a part or Assembly of a metal-cutting machine are given, depending on their material (thermal conductivity coefficient $\lambda \mathrm{W} / \mathrm{m} \times 0 \mathrm{~K}$ ) and heat exchange conditions (heat transfer coefficient $\alpha \mathrm{W} / \mathrm{m} 2 \times 0 \mathrm{~K}$ ), under which the dimension of the task can be reduced, i.e. the two-dimensional task can be considered as onedimensional without loss of solution accuracy.For example, for a column (cast iron) or machine bed, the problem can be considered as two-dimensional with a column thickness of less than 0.688 meters, and for a machine spindle (steel $40 \mathrm{X}$ ), the temperature will be uniform over its diameter, the value of which does not exceed $0.150 \mathrm{~m}$.and the temperature distribution will occur along its length and the problem will be considered as one-dimensional with heat transfer on the surface of the spindle (see Fig.2).

The thermal model of the spindle can be represented geometrically as a solid or hollow cylinder with a constant or variable cross-section, on the outer and inner surfaces of which heat exchange with the environment occurs with a heat transfer coefficient $\alpha$. In this case, each surface can have its own values of the heat transfer coefficient due to different conditions of heat exchange with the environment. For example, the heat exchange 
conditions on the surfaces of the protruding part of the spindle may differ greatly from the heat exchange conditions on the inner surface of the spindle, where heat exchange with the environment is significantly difficult. Heat supply to the spindle is surface and is carried out from various heat sources: spindle supports; drive elements; tools or parts that are fixed in the spindle and are heated due to heat generation during cutting.

Table 1. Values of the characteristic size Ld of the machine part (units), to reduce the dimension of the mathematicals task.

\begin{tabular}{|l|l|l|l|l|l|l|l|l|l|l|l|}
\hline$\lambda$ & 10 & 15 & 20 & 25 & 30 & 35 & 40 & 45 & 50 & 55 & 60 \\
\cline { 1 - 7 }$\alpha$ & & & & & & & & & & & \\
\hline 2 & 0.5 & 0.75 & 1.0 & 1.25 & 1.50 & 1.75 & 2.0 & 2.25 & 2.50 & 2.75 & 3.0 \\
\hline 4 & 0.25 & 0.38 & 0.5 & 0.63 & 0.75 & 0.88 & 1.0 & 1.13 & 1.25 & 1.36 & 1.5 \\
\hline 6 & 0.17 & 0.25 & 0.33 & 0.42 & 0.50 & 0.58 & 0.67 & 0.75 & 0.83 & 0.92 & 1.0 \\
\hline 8 & 0.13 & 0.19 & 0.25 & 0.31 & 0.38 & 0.44 & 0.50 & 0.56 & 0.63 & 0.69 & 0.75 \\
\hline 10 & 0.10 & 0.15 & 0.20 & 0.25 & 0.30 & 0.35 & 0.40 & 0.45 & 0.50 & 0.55 & 0.6 \\
\hline 12 & 0.08 & 0.14 & 0.17 & 0.21 & 0.25 & 0.29 & 0.33 & 0.38 & 0.42 & 0.46 & 0.5 \\
\hline 14 & 0.07 & 0.11 & 0.14 & 0.18 & 0.21 & 0.25 & 0.29 & 0.32 & 0.36 & 0.39 & 0.43 \\
\hline 16 & 0.06 & 0.09 & 0.13 & 0.16 & 0.19 & 0.22 & 0.25 & 0.28 & 0.31 & 0.34 & 0.38 \\
\hline 18 & 0.06 & 0.08 & 0.11 & 0.14 & 0.17 & 0.19 & 0.22 & 0.25 & 0.28 & 0.31 & 0.33 \\
\hline 20 & 0.05 & 0.07 & 0.10 & 0.13 & 0.15 & 0.18 & 0.20 & 0.23 & 0.25 & 0.28 & 0.30 \\
\hline
\end{tabular}

As a thermal model (Fig. 2) of a spindle Assembly and a ball screw Assembly, we take a cylinder of finite length $\mathrm{L}$, on the side surfaces of which heat exchange with the environment occurs with a heat exchange coefficient equal to $\alpha$, and heat is supplied to the cylinder at the places where the bearings are fixed or only through the end surfaces. The following assumptions are made:

-the problem is considered as one-dimensional and the temperature changes only along the length of the cylinder, and on each section it made constant and does not depend on radius and angle;

-thermal parameters (heat transfer coefficients, thermal conductivity and heat capacity) are functions of temperature, coordinate and time, and the heat transfer part of the end surfaces can be neglected due to the small size of their area in relation to the lateral surface of the cylinder.

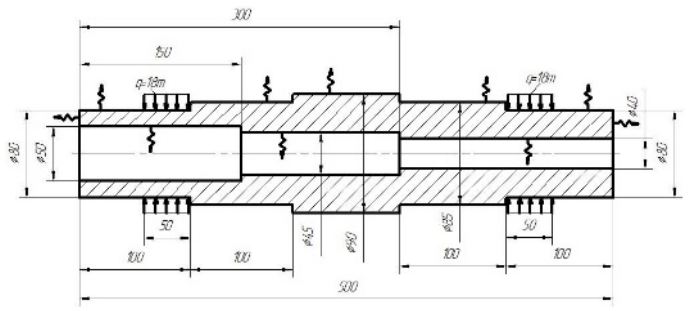

a)

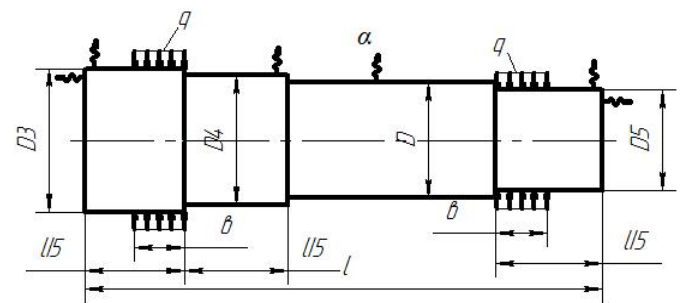

b)

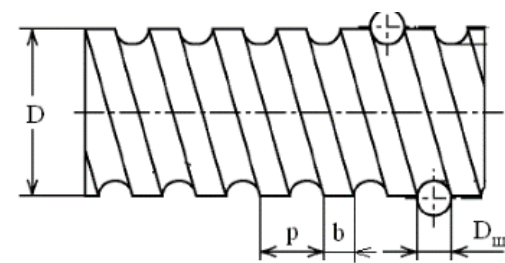

c)

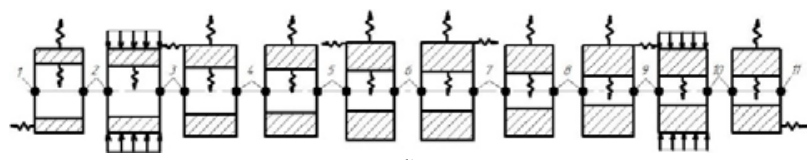

d)

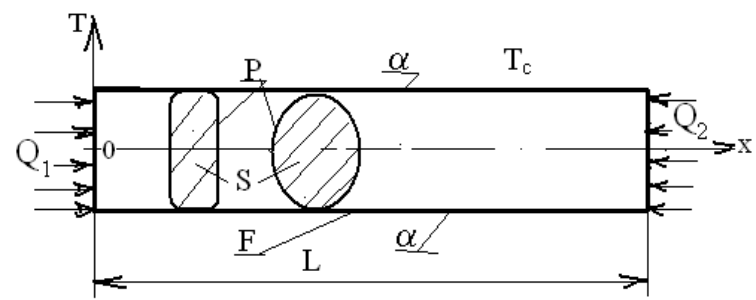

e)

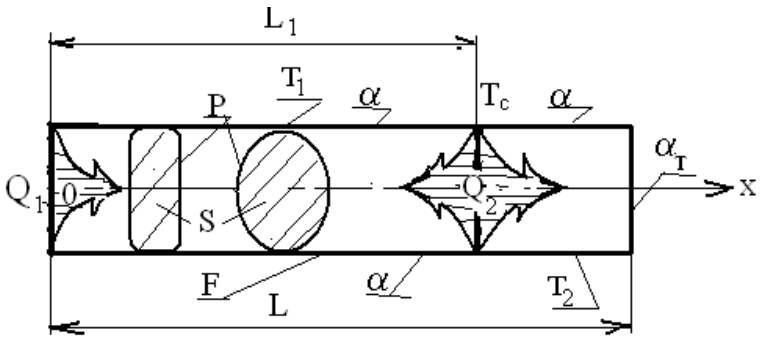

f)

Fig. 2. Thermal image of the spindle $-a$, b; parameters of the ball screw-c; thermal models for FEM-d; reduced to the cylinder-d, e.

Due to the assumptions made, this thermal model is not completely identical to the actual spindle node. However, in order for the thermal processes to be similar, it is necessary to choose such defining cylinder dimensions that the similarity criteria of Bio and Fourier are equal for both the thermal model and the actual spindle design.

When drawing up a thermal model, almost in most cases it is necessary to change (simplify or reduce to simpler forms) the geometric dimensions of real parts, as shown in Fig.2. In this case, to preserve the equivalence of conditions thermal processes can proceed in two ways.

The first is to reduce the part to a simple geometric shape while maintaining the volume of the real part and the thermal model while maintaining the same heat flow. Then in calculations it is necessary to ensure equality of the areas of the heat exchange surfaces of the thermal model Ftm and the real part Fre, and the heat exchange coefficient should be proportionally changed to the ratio of these areas. The given value, for example, of the heat flow will be equal to Qeq= Qre(Fre,/ Ftm ) and accordingly, the heat transfer coefficient of the thermal model will be equal to aeq= are(Fre,/ Ftm ). For linear 
dimensions, their values are determined for the same reasons, for example, Ltm $=$ Vre $/$ Ftm .

Second, if the lengths of the thermal model and the spindle are the same, then the diameter of the cylinder of the thermal model can be found from the equality of the Biot or Fourier criteria. In General, the most accurate value for the cylinder diameter of the thermal model will be determined by the expression:

$$
\mathrm{d}_{\mathrm{mc}}=\alpha_{\mathrm{cp}}\left(\sum \mathrm{d}_{\mathrm{i}}^{2} \mathrm{l}_{\mathrm{i}}-\sum \mathrm{d}_{\mathrm{j}}^{2} \mathrm{l}_{\mathrm{j}}\right) /\left(\sum \alpha_{\mathrm{i}} \mathrm{d}_{\mathrm{i}} \mathrm{l}_{\mathrm{i}}+\sum \alpha_{\mathrm{j}} \mathrm{d}_{\mathrm{j}} \mathrm{l}_{\mathrm{j}}+\sum \alpha_{\mathrm{ri}}\left(\mathrm{d}_{\mathrm{i}}^{2}-\mathrm{d}_{\mathrm{j}}^{2}\right)\right)
$$

where $\alpha_{\mathrm{cp}}$ is the accepted heat transfer coefficient of the thermal model or calculated from the dependence;

$$
\alpha_{\mathrm{cp}}=\left[\sum\left(\alpha_{\mathrm{i}} \mathrm{F}_{\mathrm{i}}\right)+\sum\left(\alpha_{\mathrm{Tj}} \mathrm{F}_{\mathrm{Tj}}\right)\right] /\left[\sum\left(\mathrm{F}_{\mathrm{i}}\right)+\sum\left(\mathrm{F}_{\mathrm{Tj}}\right)\right]
$$

where $\alpha_{\mathrm{i}}$ is the heat transfer coefficient of the i-th heat transfer surface Fi(internal and external), $\alpha_{\mathrm{rj}}$ is the heat transfer coefficient of the end surfaces.

In the case when it is necessary to take into account the heat transfer from the end surface instead of the length $L$ of the spindle, take its reduced length LPR

$$
L_{n p}=L+\left(\alpha_{m} / \alpha\right) \cdot(F / P)
$$

where $\mathrm{P}$ is the perimeter of a cylinder or rod of arbitrary cross-section.

It should be taken into account that the heat transfer coefficient from the screw surface is assumed to be equal to the given value:

$$
\alpha_{n p}=\alpha_{c} \cdot \sqrt{\left[1+(p / \pi D)^{2}\right]} \cdot\left[\left(\pi r_{b}+b\right) / p\right]
$$

where $\alpha_{c}$ is the heat transfer coefficient of the cylindrical surface of the screw model,$r_{b}$ is the radius of the rolling elements, $D$ is the cylinder diameter, $p$ is the screw pitch, and $b$ is the width of the screw profile pad.

For a more accurate determination of the reduced heat transfer coefficient, when considering the screw as a finned surface, the expression for calculating the $\alpha_{n p}$, based on the main provisions of the works, is written:

$$
\alpha_{n p}=\alpha_{c} \cdot\left(1+E \cdot \varepsilon \cdot \sqrt{\left[1+(p / \pi D)^{2}\right]} \cdot\left[\left(\pi r_{b}+b\right) / p\right]\right)(5)
$$

where $E$ is the coefficient of finning efficiency, and $\varepsilon$ is the coefficient of the shape of the edge profile (for a straight edge, it is equal to one).

To determine the temperature axial displacements (Fig. 3), it is necessary to solve the thermoelastic task in a quasi-static formulation, when dynamic effects during heating are not taken into account. In this formulation, the problem allows you to determine the axial deformations of both spindles and lead screws. We will consider this problem with the following assumptions: the temperature field is axisymmetric;- when heated, the thermophysical and thermomechanical parameters of the material remain constant;- Dugamel's hypothesis is valid, according to which acceleration can be ignored;-the thermoelastic state is uniaxial.
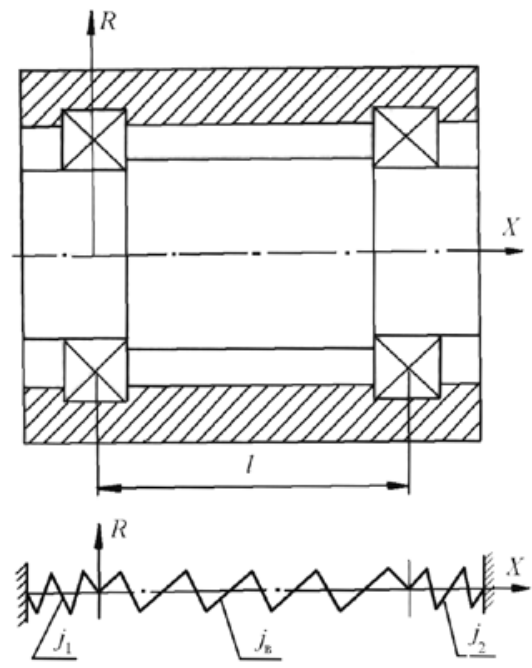

Fig.3. Typical thermoelastic model (the spindle, the lead screw) with elastic links.

Therefore, to determine the value of the thermoelastic displacement of a typical machine element (spindle or lead screw), we present it as a rod of finite length, whose quasi-thermostable connections are located only in the direction of the coordinate axes and have rigidity: the proper axial stiffness of the rod, the axial stiffness of the first and second supports . A typical thermoelastic model for this case is shown in Fig. 3. The non-stationary temperature field of the rod must also be known as the initial data for determining thermoelastic displacements at any time. To determine the nonstationary (i.e. changing in time and space) of the temperature field on the basis of the thermal model Fig.2 it is necessary to solve the heat equation with boundary conditions of the second kind:

$$
\begin{aligned}
& \partial^{2} \mathrm{~T} / \partial \mathrm{x}^{2}-\mathrm{m}^{2} \mathrm{~T}=\mathrm{a} \cdot \partial \mathrm{T} / \partial \tau ; \\
& \left.\begin{array}{ccc}
\partial T /\left.\partial x\right|_{x=0} & = & -\mathrm{Q}_{1} / F \cdot \lambda ; \\
\partial T /\left.\partial x\right|_{x=l} & = & \mathrm{Q}_{2} / F \cdot \lambda ; \\
\left.T(x, \tau)\right|_{\tau=0}= & 0
\end{array}\right\}
\end{aligned}
$$

where is the cross-sectional area of the cylinder;- values of the heat flow in the rear and front supports;coefficient inverse to the coefficient of thermal conductivity (- specific heat capacity, - specific weight of the material, - thermal conductivity of the material);heat transfer coefficient;- thermal conductivity; .

The General solution for determining the nonstationary temperature field has the form [23]: 
$T(x, \tau)=\frac{\mathrm{Q}_{2} \cdot \mathrm{K}_{\mathrm{Q}_{2}}}{F \lambda \sqrt{a} 2 \sqrt{2 b}}\left\{\sum_{n=0}^{\infty}\left[e^{-M \sqrt{2 b}} \operatorname{erfc}\left(\frac{M}{2 \sqrt{\tau}}-\sqrt{2 b \tau}\right)-e^{M \sqrt{2 b}} \operatorname{erfc}\left(\frac{M}{2 \sqrt{\tau}}+\sqrt{2 b \tau}\right)\right]+\right.$

$\left.+\sum_{n=0}^{\infty}\left[e^{-M_{1} \sqrt{2 b}} \operatorname{erfc}\left(\frac{M_{1}}{2 \sqrt{\tau}}-\sqrt{2 b \tau}\right)-e^{M_{1} \sqrt{2 b}} \operatorname{erfc}\left(\frac{M_{1}}{2 \sqrt{\tau}}+\sqrt{2 b \tau}\right)\right]\right\}+\frac{\mathrm{Q}_{1} \cdot \mathrm{K}_{\mathrm{Q}_{1}}}{F \lambda \sqrt{a} 2 \sqrt{2 b}}$

$\left\{\sum_{n=0}^{\infty}\left[e^{-U \sqrt{2 b}} \operatorname{erfc}\left(\frac{U}{2 \sqrt{\tau}}-\sqrt{2 b \tau}\right)-e^{U \sqrt{2 b}} \operatorname{erfc}\left(\frac{U}{2 \sqrt{\tau}}+\sqrt{2 b \tau}\right)\right]+\right.$

$\left.+\sum_{n=0}^{\infty}\left[e^{-U_{1} \sqrt{2 b}} \operatorname{erfc}\left(\frac{U_{1}}{2 \sqrt{\tau}}-\sqrt{2 b \tau}\right)-e^{U_{1} \sqrt{2 b}} \operatorname{erfc}\left(\frac{U_{1}}{2 \sqrt{\tau}}+\sqrt{2 b \tau}\right)\right]\right\}$

where indicated:

$$
\begin{gathered}
M=\sqrt{a}[(2 n+1) l-x], U=\sqrt{a}(2 n l-x), \\
M_{1}=\sqrt{a}[(2 n+1) l+x], \\
U_{1}=\sqrt{a}[(n+1) 2 l-x], 2 b=m^{2} / a, m^{2}=4 \alpha / \lambda d
\end{gathered}
$$

$K_{Q_{1}}, K_{Q_{2}}$ - coefficients that take into account the boundary conditions of various heat transfer on the end and other surfaces of the model among them other than the cylindrical one. For example, for different heat exchange on a part of a cylindrical surface and heat exchange on an end surface, the value will be defined as:

$$
K_{Q_{2}}=\frac{D_{m}}{1+\left(m_{1} / m_{2}\right) \cdot D_{m} \cdot \operatorname{th}\left(m_{1} \cdot L_{1}\right)}
$$

where indicated:

\begin{tabular}{|c|c|c|c|c|c|}
\hline $\mathrm{N}$ п/п & $\begin{array}{c}\alpha_{1}, \\
w / M^{2} 0 K\end{array}$ & $\begin{array}{c}\alpha_{2}, \\
w / M^{2} 0 K\end{array}$ & $\begin{array}{c}\alpha_{\mathrm{T}}, \\
\mathrm{w} / \mathrm{M}^{2} 0 \mathrm{~K}\end{array}$ & $\begin{array}{c}\mathrm{T}(0), \\
0 \mathrm{~K}\end{array}$ & $\begin{array}{c}\mathrm{T}(0.4), \\
0 \mathrm{~K}\end{array}$ \\
\hline 1 & 25 & 25 & 0 & 15.02 & 21.87 \\
\hline 2 & 25 & 25 & (Lпр) & 14.66 & 20.54 \\
\hline 3 & 25 & 25 & 25 & 14.66 & 20.53 \\
\hline 4 & 25 & 50 & 0 & 13.85 & 17.46 \\
\hline 5 & 25 & 50 & 50 & 13.42 & 15.85 \\
\hline 6 & 125 & 25 & 0 & 4.51 & 10.67 \\
\hline 7 & 125 & 50 & 0 & 4.48 & 9.30 \\
\hline 8 & 25 & 25 & 500 & 13.19 & 14.99 \\
\hline 9 & 25 & 50 & 500 & 12.41 & 12.07 \\
\hline 10 & 25 & 25 & 1500 & 12.53 & 12.51 \\
\hline 11 & 325 & 50 & 1500 & 2.66 & 5.13 \\
\hline
\end{tabular}

$$
\begin{gathered}
D_{m}=\left[K_{m} \cdot \exp \left(2\left(L-L_{1}\right) \cdot m_{2}\right)+1\right] /\left[K_{m} \cdot \exp \left(2\left(L-L_{1}\right) \cdot m_{2}\right)-1\right] \\
K_{m}=\left[m_{2}+\left(\alpha_{m} / \lambda\right)\right] /\left[m_{2}-\left(\alpha_{m} / \lambda\right)\right]
\end{gathered}
$$

Table 2. Comparative data for determining temperatures by (8) for different $K_{Q_{1}}, K_{Q_{2}}$.

\section{Non-stationary deformations}

To determine the temperature deformations that are caused by an axisymmetric non-stationary temperature field, it is necessary to solve the General equation in displacements for the thermoelastic model (Fig.3.) with boundary conditions determined by the elastic state of the rod (cylinder) and fixing in the locations of the supports: Equations should be centred and should be numbered with the number on the right-hand side.

$$
\begin{gathered}
\frac{\partial^{2} U}{\partial x^{2}}=\beta \cdot \frac{\partial T}{\partial x} \\
\varepsilon_{x x} \cdot E-\beta \cdot E \cdot T=\sigma_{x x} \text { при } x=0 \\
\varepsilon_{x x} \cdot E-\beta \cdot E \cdot T=-\sigma_{x x} \text { при } x=L
\end{gathered}
$$

The General expression for determining axial offsets in an arbitrary cross section is [24]:

$$
\begin{aligned}
& U(x, \tau)=\int_{0}^{x} \beta \cdot T(x, \tau) \cdot d x-\frac{x \cdot j_{\Sigma}}{E \cdot F} \int_{0}^{L} \beta \cdot T(x, \tau) \cdot d x-\frac{j_{\Sigma}}{j_{1}} \int_{0}^{L} \beta \cdot T(x, \tau) \cdot d x= \\
& =\int_{0}^{x} \beta \cdot T(x, \tau) \cdot d x-j_{\Sigma} \cdot\left(j_{1}^{-1}+\frac{x}{E \cdot F}\right) \cdot \int_{0}^{L} \beta \cdot T(x, \tau) \cdot d x
\end{aligned}
$$

where $\beta$ - coefficient of thermal expansion of the material, $E$ - modulus of elasticity.

For boundary conditions when there is an initial gap $\delta_{0}$ in the support, and there is a pre-stressed state along the axis, i.e., when the spindle or lead screw is prestretched (compressed) by force $P_{n p}$, the generalized expression for estimating the axial displacement at an arbitrary time and in an arbitrary cross section will take the form:

$U(x, \tau)=\int_{0}^{x} \beta T(x, \tau) d x-\frac{x \cdot P_{n p}}{E F}-j_{\Sigma}\left(j_{1}^{-1}+\frac{x}{E F}\right)\left[\int_{0}^{L} \beta T(x, \tau) d x-\frac{x \cdot P_{n p}}{E F}-\delta_{0}\right]$

The expression (13) allows you to determine the thermal displacements of bodies that can be reduced to the considered thermal model. When considering the thermal displacements of the spindle, we are usually interested in the amount of displacement of its end face . We transform the expression (13) by dividing its left and right parts by the same value equal to the value of the thermal displacement of the spindle free from external loads, i.e., when the spindle supports do not interfere with its displacement, and as a result we get:

$$
\begin{aligned}
& \frac{U(L, \tau)}{\int_{0}^{L} \beta T(x, \tau) d x}=\frac{j_{\Sigma}}{j_{2}}-\frac{j_{\Sigma}}{j_{2}} \cdot \frac{L \cdot P_{n p}}{E F \int_{0}^{L} \beta T(x, \tau) d x}+\frac{j_{\Sigma}^{L} \cdot\left(j_{1}^{-1}+\frac{L}{E F}\right) \delta_{0}}{\int_{0}^{L} \beta T(x, \tau) d x}= \\
& =\frac{j_{\Sigma}}{j_{2}}-\frac{j_{\Sigma}}{j_{2}} \cdot \frac{j_{n p}}{j_{6}}+j_{\Sigma} \cdot\left(j_{1}^{-1}+j_{6}^{-1}\right) \cdot K_{0}=K_{j} \cdot \int_{0}^{L} \beta T(x, \tau) d x
\end{aligned}
$$

where indicated: 


$$
\begin{gathered}
K_{j}=\frac{j_{\Sigma}}{j_{2}} \cdot\left(1-K_{n p}-K_{0}\right)+K_{0}=\left[\frac{j_{\Sigma}}{j_{2}} \cdot\left(1-K_{n p}\right)+K_{0} \cdot\left(1-K_{n p}\right)\right] \\
K_{n p}=j_{n p} / j_{B}, j_{B}=E F / L, \\
j_{n p}=P_{n p} / \int_{0}^{L} \beta T(x, \tau) d x \\
j_{\Sigma}=\left[j_{1}^{-1}+j_{2}^{-1}+j_{B}^{-1}\right], K_{0}=\delta_{0} / \int_{0}^{L} \beta \cdot T(x, \tau) \cdot d x
\end{gathered}
$$

The range of values is from 0 to 1 , and the limit values correspond to the following ratios of stiffness values:

$$
\begin{gathered}
j_{2}=0 \Rightarrow K_{j}=1 ; j_{2}=\infty \Rightarrow K_{j}=0 \\
j_{1}=0 \Rightarrow K_{j}=0 ; \quad j_{1}=\infty \Rightarrow K_{j}=j_{b} /\left(j_{b}+j_{2}\right)
\end{gathered}
$$

In order to get an explicit solution for determining non-stationary temperature displacements, it is necessary to substitute the value of the function in the expression (8).

Then, the final solution will be obtained when all the components of the expression $(13,14)$ are sequentially integrated. Subintegral expressions are structurally similar functions. As an example, let's integrate one of the same type of functions:

$\Omega=\int_{0}^{X} e^{-U \sqrt{2 b}} \operatorname{erfc}\left[\frac{U}{2 \sqrt{\tau}}-\sqrt{2 b \tau}\right]=\int_{0}^{X} \exp [-\sqrt{2 b}(2 n l+\xi) \sqrt{a}] \operatorname{erfc}\left[\frac{\sqrt{a}(2 n l+\xi)}{2 \sqrt{\tau}}-\sqrt{b \tau}\right] d \xi$

Perform the following conversions:

$$
e^{-\sqrt{2 b a(2 n l+\xi)}}=\exp [-\xi \sqrt{2 b a}] \cdot \exp [-\sqrt{2 b a} \cdot 2 n l]
$$

$$
\frac{\sqrt{a}(2 n l+\xi)}{2 \sqrt{\tau}}-\sqrt{2 b \tau}=\frac{\xi \sqrt{a}}{2 \sqrt{\tau}}+\frac{\sqrt{a} 2 n l}{2 \sqrt{\tau}}-\sqrt{2 b \tau}
$$

Substituting in (17), we get:

Denote $2 n l \sqrt{a}-\tau \sqrt{8 b}=A$ and $A+\xi \sqrt{a}=\mu$ introduce new variables:

$$
\begin{gathered}
u=\operatorname{erfc}\left(\frac{\mu}{2 \sqrt{\tau}}\right) \\
d u=-\frac{\sqrt{a}}{\sqrt{\pi \tau}} \exp \left(-\frac{\mu}{4 \tau}\right) d \xi
\end{gathered}
$$

$$
\begin{gathered}
v=-\frac{1}{\sqrt{2 b a}} \cdot \exp (-\zeta \cdot \sqrt{2 b a}) \\
d v=-\frac{1}{\sqrt{2 b a}} d[\exp (-\xi \sqrt{2 b a})]
\end{gathered}
$$

Then the expression (15) will look like:

$$
\begin{aligned}
& \Omega=\exp [-\sqrt{2 b a} \cdot 2 n l]\left\{\left(\frac{1}{\sqrt{2 b a}}\right) \exp (-\xi \sqrt{2 b a}) \times \operatorname{erfc}\left(\frac{\mu}{2 \sqrt{\tau}}\right)-\right. \\
& -\int_{0}^{x} \exp \left[-\left(\frac{\mu}{4 \tau}+\xi \sqrt{2 b a}\right)\right] \cdot\left(\frac{\sqrt{a}}{\sqrt{\pi \tau \sqrt{2 b a}}}\right) d \xi
\end{aligned}
$$

Transform the newly obtained integrand:

$\exp \left[-\left(\frac{\mu^{2}}{4 \tau}+\xi \sqrt{2 b a}\right)\right]=\exp \left[-\frac{\left(\xi \sqrt{a}+A_{1}\right)^{2}}{4 \tau}\right] \exp \left(\frac{A^{2}-A^{2}}{4 \tau}\right)$

where indicated $A_{1}=A+2 \tau \sqrt{2 b}=2 n l \sqrt{a}$. As a result, the expression is written as follows:

$$
\begin{aligned}
& \Omega=\exp (-2 n l \sqrt{2 b a}) \frac{1}{\sqrt{2 b a}}\left\{-\left.\exp (-\xi \sqrt{2 b a}) \operatorname{erfc}\left(\frac{\mu}{2 \sqrt{\tau}}\right)\right|_{0} ^{x}-\right. \\
& \left.--\frac{\sqrt{a}}{\sqrt{\pi \tau}} \exp \left(\frac{A^{2}-A^{2}}{4 \tau}\right) \int_{0}^{x} \exp \left[\frac{\left(\xi \sqrt{a}+A_{1}\right)^{2}}{4 \tau}\right] d \xi\right\}
\end{aligned}
$$

We introduce new variable integration limits from the given boundary conditions defined by the expressions:

выражениями:

$$
\begin{gathered}
\gamma=\frac{\xi \sqrt{a+A_{1}}}{2 \sqrt{\tau}} ; \xi=\gamma \frac{2 \sqrt{\tau}}{\sqrt{a}}-\frac{A_{1}}{\sqrt{a}} ; \quad d \xi=\frac{2 \sqrt{\tau}}{\sqrt{a}} d \gamma . \\
\xi=0 \quad \gamma=\frac{A_{1}}{2 \sqrt{\tau}} ; \quad \xi=x \quad \gamma=\frac{x \sqrt{a+} A_{1}}{2 \sqrt{\tau}} .
\end{gathered}
$$

Substituting new variables in the expression and performing integration within the new limits, we get:

$$
\begin{aligned}
& \Omega=\exp (-2 n l \sqrt{2 b a}) \frac{1}{\sqrt{2 b a}}\left\{-\left.\exp (-\xi \sqrt{2 b a}) \operatorname{erfc}\left(\frac{\mu}{2 \sqrt{\tau}}\right)\right|_{0} ^{X}-\right. \\
& -\frac{\sqrt{a}}{\sqrt{\pi \tau}} \exp \left(\frac{A^{2}-A}{4 \tau}\right) \underset{\frac{A_{1}}{2 \sqrt{\tau}}}{\int^{\frac{z \sqrt{A}}{2}+A_{1}}} \exp (-\gamma) \frac{2 \sqrt{\tau}}{\sqrt{a}} d \gamma
\end{aligned}
$$


Sequentially defining the value of the function $\mathrm{x}$ within the integration, we find the expression explicitly:

$$
\begin{aligned}
& \Omega=\exp (-2 n l \sqrt{2 b a}) \frac{1}{\sqrt{2 b a}}\left\{\left[-\exp (-x \sqrt{2 b a}) \operatorname{erfc}\left(\frac{x \sqrt{a+A}}{2 \sqrt{\tau}}\right)+\right.\right. \\
& \left.+\operatorname{erfc}\left(\frac{A}{2 \sqrt{\tau}}\right)\right]-\exp \left(\frac{A^{2}-A^{2}}{4 \tau}\right)\left[\operatorname{erf}\left(\frac{x \sqrt{a}+A 1}{2 \sqrt{\tau}}\right)-\right. \\
& \left.\left.-\operatorname{erf}\left(\frac{A 1}{2 \sqrt{\tau}}\right)\right]\right\}=\delta_{X}\left(-U, A, A_{1}\right)
\end{aligned}
$$

Performing similar transformations with all the components of the expression, we find an explicit solution to the problem of determining non-stationary thermoelastic displacements for the thermal model shown in Fig.3.

$\delta(x, \tau)=\frac{\beta q_{1}}{F \lambda 2 \sqrt{2 b a}}\left\{\sum\left[\delta_{x}\left(-U, A, A_{1}\right)-\delta_{x}\left(U, A_{2}, A_{3}\right)\right]+\sum\left[\delta_{x}\left(-U_{1}, A^{\prime}, A_{1}^{\prime}\right)-\delta_{x}\left(U_{1} A_{2}^{\prime}, A_{3}^{\prime}\right)\right]\right\}+$ $+\frac{\beta q_{2}}{F \lambda 2 \sqrt{2 b a}}\left\{\sum\left[\delta_{x}\left(-M, B^{\prime}, B_{1}^{\prime}\right)-\delta_{x}\left(M, B_{2}^{\prime}, B_{3}^{\prime}\right)\right]+\sum\left[\delta_{x}\left(-M_{1}, B, B_{1}\right)-\delta_{x}\left(M_{1}, B_{2}, B_{3}\right)\right]\right\}-j_{\Sigma}\left(\frac{x}{E F}+\right.$ $\left.+\frac{1}{\mathrm{j}_{1}}\right) \frac{\beta \mathrm{q}_{1}}{\mathrm{~F} \lambda 2 \sqrt{2 \mathrm{ba}}}\left\{\sum\left[\delta_{1}\left(-\mathrm{U}, \mathrm{A}, \mathrm{A}_{1}\right)-\delta_{1}\left(\mathrm{U}, \mathrm{A}_{2}, \mathrm{~A}_{3}\right)\right]+\sum\left[\delta_{1}\left(-\mathrm{U}_{1}, \mathrm{~A}^{\prime}, \mathrm{A}_{1}^{\prime}\right)-\delta_{1}\left(\mathrm{U}_{1}, \mathrm{~A}_{2}^{\prime}, \mathrm{A}_{3}^{\prime}\right)\right]\right\}$ $+\frac{\beta q_{2}}{F \lambda 2 \sqrt{2 b a}}\left\{\sum\left[\delta_{1}\left(-M, B^{\prime}, B_{1}^{\prime}\right)-\delta_{1}\left(M, B_{2}^{\prime}, B_{3}^{\prime}\right)\right]+\sum\left[\delta_{1}\left(-M_{1}, B, B_{1}\right)-\delta_{1}\left(M_{1}, B_{2}, B_{3}\right)\right]\right\} ;$

where indicated:

$$
\begin{aligned}
& U=\sqrt{a}(2 n l+x) ; \quad U_{1}=\sqrt{a}[2 l(n+1)-x] ; \\
& M_{1}=\sqrt{a}[l(2 n+1)+x] ; \quad M=\sqrt{a}[l(2 n+1)-x] ; \\
& A=2 n l \sqrt{a}-2 \tau \sqrt{2 b} ; \quad A_{1}=A+2 \tau \sqrt{2 b ;} \\
& A^{\prime}=2 l(n+1) \sqrt{a}-2 \tau \sqrt{2 b} ; \quad A_{1}^{\prime}=A^{\prime}+2 \tau \sqrt{2 b} ; \\
& B=l(2 n+1) \sqrt{a}-2 \tau \sqrt{2 b ;} \quad B_{1}=B+2 \tau \sqrt{2 b} ; \\
& B^{\prime}=l(2 n+1) \sqrt{a}-2 \tau \sqrt{2 b} ; \quad B_{1}^{\prime}=B^{\prime}+2 \tau \sqrt{2 b} ; \\
& A_{2}=2 n l \sqrt{a}+2 \tau \sqrt{2 b} ; \quad A_{3}=A_{2}-2 \tau \sqrt{2 b} ; \\
& A_{2}^{\prime}=2 l(n+1) \sqrt{a}+2 \tau \sqrt{2 b} ; \quad A_{3}^{\prime}=A_{2}^{\prime}-2 \tau \sqrt{2 b a} ; \\
& B_{2}=l(2 n+1) \sqrt{a}+2 \tau \sqrt{2 b} ; \quad B_{3}=B_{2}-2 \tau \sqrt{2 b a} ; \\
& B_{2}^{\prime}=l(2 n+1) \sqrt{a}+2 \tau \sqrt{2 b} ; \quad B_{3}=B_{2}^{\prime}-2 \tau \sqrt{2 b} ;
\end{aligned}
$$

The expression includes functions $\delta_{x}$ and $\delta_{1}$ that characterize the values obtained after defining the corresponding integral expressions with integration boundaries from 0 to $x$ and from 0 to $l$, and the parameters on which they depend are indicated in parentheses/Here are several components of the expression (23):

$$
\begin{aligned}
& \delta_{x}\left(-U, A, A_{1}\right)=\frac{1}{m} \exp (-2 m n l)\left\{\left[\operatorname{erfc}\left(\frac{A}{2 \sqrt{\tau}}\right)-e^{-m x} \cdot \operatorname{erfc}\left(\frac{A+x \sqrt{a}}{2 \sqrt{\tau}}\right)\right]-\right. \\
& \left.-\exp \left(\frac{A_{1}^{2}-A^{2}}{4 \tau}\right)\left[\operatorname{erf}\left(\frac{A_{1}+x \sqrt{a}}{2 \sqrt{\tau}}\right)-\operatorname{erf}\left(\frac{A_{1}}{2 \sqrt{\tau}}\right)\right]\right\}
\end{aligned}
$$

$$
\begin{aligned}
& \delta_{X}\left(U_{1}, A_{2}^{\prime}, A_{3}^{\prime}\right)=\frac{1}{m} \exp \left[2 m ( n + 1 ) l \left[\left\{\left[e^{-m x} \cdot \operatorname{erfc}\left(\frac{A_{2}^{\prime}-x \sqrt{a}}{2 \sqrt{\tau}}\right)-\operatorname{erfc}\left(\frac{A_{2}^{\prime}}{2 \sqrt{\tau}}\right)\right]+\right.\right.\right. \\
& \left.+\exp \left(\frac{A_{3}^{\prime 2}-A_{2}^{\prime 2}}{4 \tau}\right)\left[\operatorname{erf}\left(\frac{x \sqrt{a-A_{3}^{\prime}}}{2 \sqrt{\tau}}\right)+\operatorname{erf}\left(\frac{A_{3}^{\prime}}{2 \sqrt{\tau}}\right)\right]\right\} \\
& \delta_{l}\left(M, B_{2}^{\prime}, B_{3}^{\prime}\right)=\frac{1}{m} \exp [m(2 n+1) l]\left\{\left[e^{-m l} \cdot \operatorname{erfc}\left(\frac{B_{2}^{\prime}-l \sqrt{a}}{2 \sqrt{\tau}}\right)-\operatorname{erfc}\left(\frac{B_{2}^{\prime}}{2 \sqrt{\tau}}\right)\right]+\right. \\
& \left.+\exp \left(\frac{B_{3}^{\prime 2}-B_{2}^{\prime 2}}{4 \tau}\right)\left[\operatorname{erf}\left(\frac{l \sqrt{a}-B_{3}^{\prime}}{2 \sqrt{\tau}}\right)+\operatorname{erf}\left(\frac{B_{3}^{\prime}}{2 \sqrt{\tau}}\right)\right]\right\}
\end{aligned}
$$

A stationary solution to the problem of determining thermoelastic displacements can be found from the expression for the value $\tau \rightarrow \infty$. Obviously, you need to find the limits of the functions $\delta_{x}, \delta_{l}$ included in the expression. As an example, we define a number of them:

$$
\begin{aligned}
& \lim \left[\delta_{x}\left(-U, A, A_{1}\right]=\lim _{\tau \rightarrow \infty} \frac{1}{m} \exp (-2 m n l)\{[\operatorname{erfc}(-\infty)-\right. \\
& \left.-\exp (-m x) \operatorname{erfc}(-\infty)]-\exp \left(\frac{A_{1}^{2}-A^{2}}{4 \tau}\right)[\operatorname{erf}(0)-\operatorname{erf}(0)]\right\}= \\
& ==-\frac{2}{m} \exp (-m n l)[1-\exp (-m x)]
\end{aligned}
$$

$\lim _{\tau \rightarrow \infty}\left[\delta_{x}\left(U, A_{2}, A_{3}\right)=\lim _{\tau \rightarrow \infty} \frac{1}{m} \exp (2 m n l)\{\exp (m x)[\operatorname{erfc}(\infty)-\right.$ $-\operatorname{erfc}(\infty)]\}=0$

Find the limit of the expression function for a multiplier $Q_{1}$ :

$$
\begin{aligned}
& \frac{\beta Q_{1}}{2 F \lambda \sqrt{2 b a}} \frac{2}{m}\left\{\sum\left[\exp (-2 m n l)\left(1-e^{-m x}\right)\right]+\sum[\exp [-2 m(n+1) l] \times\right. \\
& \left.\left.\times\left(e^{m x}-1\right)\right]\right\}=\frac{\beta Q_{1}}{F \lambda m^{2}}\left[\frac{\left(1+c t h(m l)\left(1-e^{-m x}\right)\right.}{2}+\frac{\left(c t h(m l)-1\left(e^{m x}-1\right)\right.}{2}\right]= \\
& =\frac{\beta Q_{1}}{F \lambda m^{2}}\left[1-\frac{\operatorname{Shm}(l-x)}{\operatorname{Sh}(m l)}\right]
\end{aligned}
$$

Similarly, we define the limit of the expression function for a multiplier $Q_{2}$ :

$$
\begin{aligned}
& \frac{\beta Q_{2}}{F \lambda m^{2}}\left\{\sum \operatorname { e x p } \left[-m(2 n+1) l\left(e^{m x}-1\right)+\sum \exp [-m(2 n+1) l] \times\right.\right. \\
& \left.\times\left(1-e^{-m x}\right)\right\}=\frac{\beta Q_{2}}{F \lambda m^{2}}\left[\frac{1}{2} \frac{1}{S h(m l)}\left(e^{m x}-1\right)+\frac{1}{2} \frac{1}{S h(m l)}\left(1-e^{-m x}\right)\right]= \\
& =\frac{\beta Q_{2}}{F \lambda m^{2}} \frac{\operatorname{Sh}(m x)}{\operatorname{Sh}(m l)}
\end{aligned}
$$

Then it is possible to determine the temperature displacements of an arbitrary cross-section of the lead screw from the following expression, which fully 
corresponds to the solution in the stationary formulation of the problem [9]:

$$
\delta(x)=\frac{\beta}{F \cdot \lambda \cdot m^{2}}\left\{Q_{2} \frac{S h(m x)}{S h(m l)}-Q_{1}\left[1-\frac{S h m(l-x)}{S h(m l)}\right]\right\}-j_{\Sigma} \cdot\left(j_{1}^{-1}+\frac{x}{E \cdot F}\right) \cdot \frac{\beta \cdot\left(Q_{1}+Q_{2}\right)}{F \cdot \lambda \cdot m^{2}}
$$

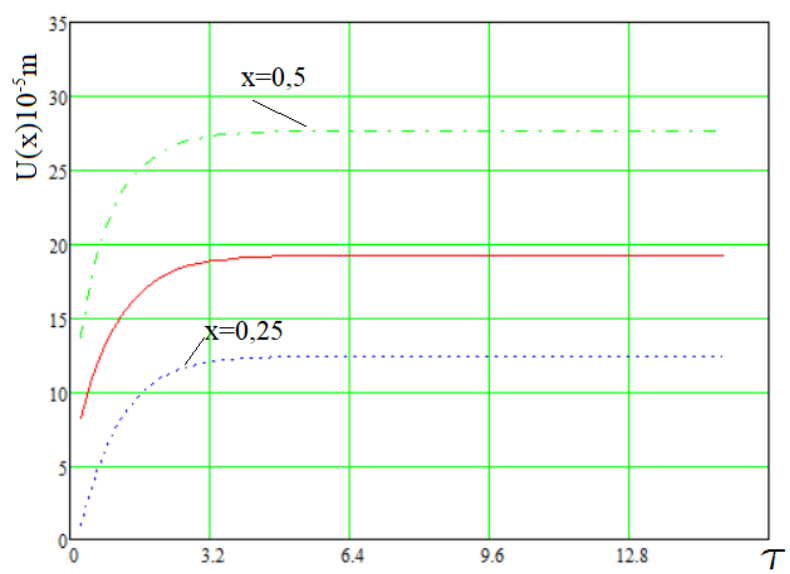

Fig. 4. The calculated values displacement.(23)

\section{Conclusion}

In this work, the temperature displacements of rod-type heat-active elements were considered for possible variants of their quasi-thermostable bonds, and expressions were obtained for describing and evaluating their values. This type is most typical for both spindle units and running screws.

In many cases of evaluating the thermal behavior of machine parts and assemblies, when their shape differs from the rod type, one can also use rod models, in which these parts are given according to criteria of similarity of processes, geometric similarity, depending on the goals and methods of solving the problem.

\section{References}

1. J. Mayr, at al, CIRP Annals - Manufacturing Technology, 61, 771-791 (2012)

2. Y. Yto, Thermal deformation in Machine tools. (McGraw-Hill, 2010)

3. A.P. Kuznetsov, Russian Engineering Research, 37(3), 171-179 (2017) https://doi.org/10.3103/S1068798X17030157

4. A.P. Kuznetsov, Stankoinstrument,. 3, 30-41 (2020)

5. S. Mekid, Introduction to precision machine design and error assessment (CRC Press, 2009)

6. A.P. Kuznetsov, H.-J. Koriath, The Conference on Thermal Issues in Machine Tools. Proceedings CIRP sponsored conference, Verlag Wissenschaftliche Scripten, Dresden, 47-60 (2018)

7. W. Xian, Hindawi Mathematical Problems in Engineering, 2018

(2018)
8. M. Mares, O. Horejs, L. Havlík, Precision Engineering, 66, 21-30 (2020) https://doi.org/10.1016/j.precisioneng.2020.06.010

9. E. Abele, Y. Altintas, C. Brecher, CIRP Annals Manufacturing Technology, 59(2), 781-802 (2010)

10. Y. Li, et al, Int J Adv Manuf Technol., 72, 14151427 (2014)

11. Y. Li, et al, Int J Mach Tools Manuf., 95, 20-38 (2015)

12. J. Zapłata, M. Pajor, Precision Engineering, 60, 160166 (2019)

https://doi.org/10.1016/j.precisioneng.2019.07.011

13. J. Jedrzejewski et al, Journal of Machine Engineering, 19(4), 27-41 (2019)

14. Z.C. Du, S.Y. Yao, J.G. Yang, Int. J. Precis. Eng. Manuf., 16, 1571-1581 (2015)

15. H.R. Cao, X.W. Zhang, X.F. Chen, Int J. Mach Tools Manuf., 112, 21-52 (2016)

16. Li. Sun, et al, Int J Adv Manuf Technol., 88, 12671277 (2017)

17. C. Ma, et al, Int J Adv Manuf Technol., 89, .30713085 (2017)

18. F. Tan, et al, The International Journal of Advanced Manufacturing Technology, 94, 2861-2874 (2018)

19. Y. Li, et al, The International Journal of Advanced Manufacturing Technology, 99, 1341-1349 (2018)

20. H. Zhou, et al, Procedia Manufacturing, 26, 11371146 (2018)

21. G. Fu, H. Gong, H. Gao, International Journal of Advanced Manufacturing Technology, 105, 20392055 (2019)

22. Q. Yin, et al,The International Journal of Advanced Manufacturing Technology, 101, 1699-1713 (2019)

23. A.P. Kuznetsov, Methods for evaluating thermal deformations of metal-cutting machines and ways to reduce them (M., Niimash, 1983)

24. A.P. Kuznetsov, Thermal processes in machine tools (M.: Technosphere, 2019) 